\title{
Descending necrotizing mediastinitis secondary to a retropharyngeal abscess
}

\author{
M. Takao*, M. Ido*, K. Hamaguchi**, H. Chikusa', S. Namikawa*, M. Kusagawa*
}

Descending necrotizing mediastinitis secondary to retropharyngeal abscess. M. Takao, M. Ido, K. Hamaguchi, H. Chikusa, S. Namikawa, M. Kusagawa. OERS Journals Ltd 1994. ABSTRACT: A rare case of descending necrotizing mediastinitis (DNM) secondary to a nontraumatic retropharyngeal abscess is reported. Even in the era of antibiotics, the mortality of DNM is still around $40 \%$.

In spite of drainage of a localized neck abscess, and the administration of systemic antibiotics, the retropharyngeal abscess extended to the pericardial and pleural cavities. Several drainage procedures and thoracotomies were performed to treat the bilateral empyemas and purulent pericarditis. The patient was discharged on hospital day 52. Computed tomography was used to follow the progression of disease, and assess the efficacy of treatment.

DNM is a very aggresive form of mediastinitis. The importance of proper mediastinal drainage, as well as the systemic administration of antibiotics, must be emphasized.

Eur Respir J., 1994, 7, 1716-1718.

\author{
Depts of *Thoracic and Cardiovascular \\ Surgery, ** Otolaryngology and ${ }^{\dagger}$ Emergency \\ Medicine and Intensive Care, Mie University \\ School of Medicine, Edobashi, Tsu, Mie, \\ Japan. \\ Correspondence: M. Takao \\ Dept of Thoracic and Cardiovascular Surgery \\ Mie University School of Medicine \\ 2-174 Edobashi \\ Tsu \\ Mie 514 Japan \\ Keywords: Mediastinitis \\ retropharyngeal abscess
}

Received: May 281993

Accepted after revision February 141994

\section{Case report}

The incidence of nontraumatic retropharyngeal abscess has been low since the introduction of antibiotics. However, the mortality remains high because of the occurrence of associated lethal complications, such as acute upper airway obstruction, aspiration pneumonia, jugular thrombophlebitis, carotid artery haemorrhage, and descending necrotizing mediastinitis (DNM) [1]. DNM is one of the most lethal forms of mediastinitis, even in the era of antibiotics [2]. Because the diagnosis of DNM is frequently delayed, cervical drainage of the original site of the infection, as well as the upper mediastinum is often insufficient [3]. In addition to the administration of antibiotics, directed both at aerobic and anaerobic organisms, early aggressive surgical drainage is essential.

a)

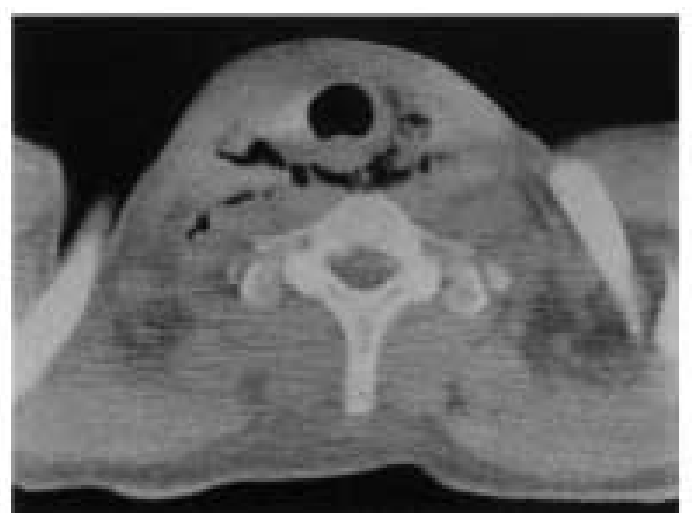

A 54 year old male was admitted to the Mie University Hospital with a 3 day history of sore throat, associated with swelling of the anterior neck and trismus. Intraoral examination revealed an erythematous bulging of the left posterior pharyngeal wall, without evidence of trauma. The tonsils and vocal cords appeared normal. Computed tomography (CT) of the neck and chest revealed collections of air extending from the pretracheal, perivascular and retrovisceral spaces down to the level of tracheal bifurcation (fig. 1).

On admission, a retropharyngeal abscess was incised and drained. Enterobacter cloacae and Candida albicans

b)

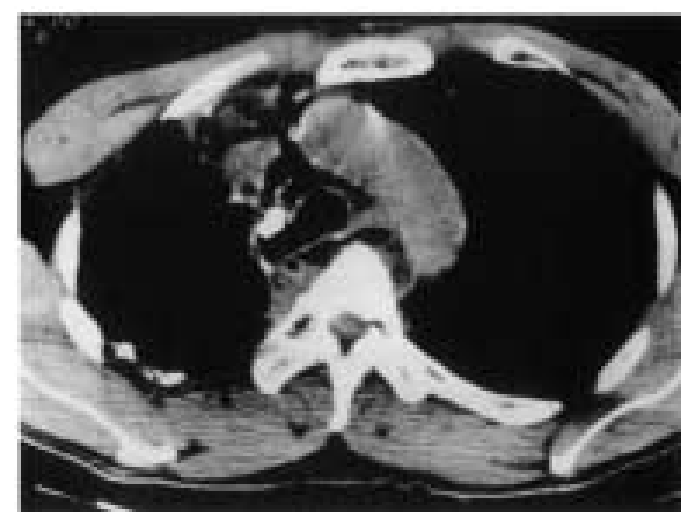

Fig. 1. - Computed tomography (CT) on the day of admission revealed a mediastinal abscess with a collection of air: a) in the pretracheal and retropharyngeal spaces; and b) extending down to the level of the tracheal bifurcation. 
a)

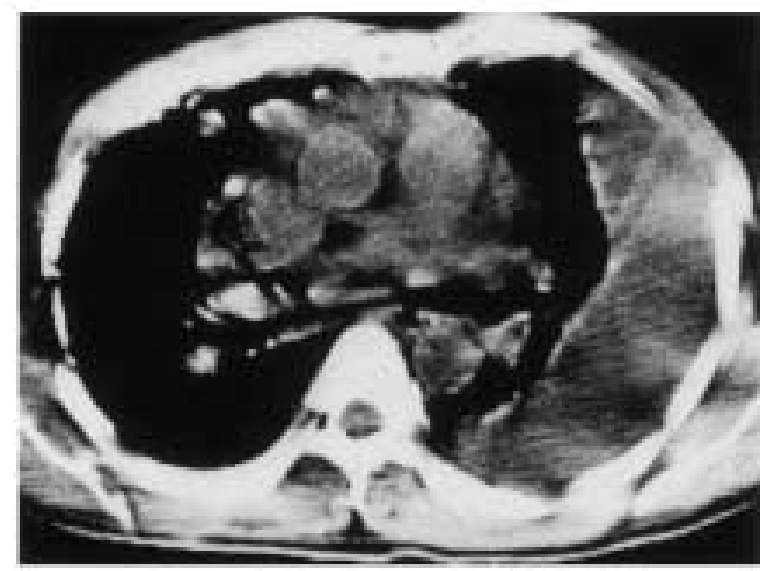

b)

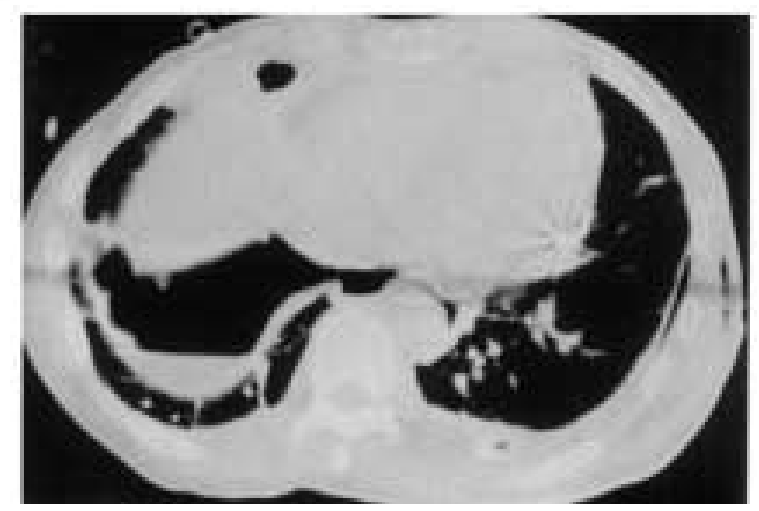

Fig. 2. - a) Computed tomography (CT) on hospital day 11 revealed descending necrotizing mediastinitis (DNM) eroding into the left pleural and pericardial cavities. b) CT on hospital day 18 revealed a rightsided empyema with an air-fluid level.

were cultured from the wound. One day postoperatively, the patient reported back pain, with relief of pharyngalgia and trismus. On hospital day 4, because of worsening anterior neck swelling, cervicomediastinal drainage was performed via a transverse cervical neck skin incision. Pus was discovered between the anterior cervical muscles and the thyroid gland, and beneath the sternomastoid muscles, along the trachea, into the upper mediastinum. Penrose drains were placed into these spaces to keep the wounds open. Infection with anaerobic bacteria was suspected, because of the gas formation and foul smell of the pus. Systemic antibiotics were intravenously administered (cefotiam $3 \mathrm{~g} \cdot \mathrm{day}^{-1}$ in three doses, and clindamycin $1.2 \mathrm{~g} \cdot \mathrm{day}^{-1}$ in two doses). The abscess cavities were irrigated with saline containing tobramycin $\left(0.2 \mathrm{~g} \cdot l^{-1}\right)$.

However, the condition of the patient deteriorated on hospital day 11, with signs of high fever, hypoxaemia (arterial oxygen tension $\left(\mathrm{PaO}_{2}\right) 7.1 \mathrm{kPa}$ with $4 \mathrm{l} \cdot \mathrm{min}^{-1} \mathrm{O}_{2}$ via nasal cannulae) and hypercapnia arterial carbon dioxide tension $\left(\mathrm{PaCO}_{2} 6.2 \mathrm{kPa}\right)$. The patient was intubated and artificially ventilated. CT of the chest revealed large pleural and pericardial collections (fig. 2a). Tube drainage of the left pleural and pericardial cavities was performed. E. cloacae and C. albicans, respectively, were cultured from the collections. A second cervical exploration was performed, which revealed no abscess around the upper trachea. The wound was packed with iodoform-gauze.

Irrigation of the left pleural and pericardial cavities was performed twice a day with saline containing amikacin $\left(0.2 \mathrm{~g} \cdot l^{-1}\right)$. An abscess developed in the right pleural cavity on hospital day 18 (fig. 2b). On hospital day 20 the right thoracic abscess cavity was decorticated and drained through a right posterolateral thoracotomy. $E$. cloacae was cultured from the abscess. A communication between the right and left pleural cavities was confirmed.

Intensive irrigation of all abscesses was performed four times a day, with $500 \mathrm{ml}$ saline containing $0.1 \mathrm{~g}$ of amikacin. Drainage eventually decreased, and surveillance cultures remained negative. The last drainage tube was removed on hospital day 43. Fever did not recur, and the patient was discharged on hospital day 52 . Endoscopic examination at discharge showed no evidence of injury to the oesophagus.

\section{Discussion}

Most cases of mediastinitis follow a median sternotomy or oesophageal perforation. Mediastinitis following a suppurative deep neck infection, descending necrotizing mediastinitis (DNM), is rare, and is associated with a poor prognosis $[2,3]$. Mortality is reported to be $43 \%$ even in the era of antibiotics [2].

Of the 43 cases of DNM reported since 1960, 25 were caused by odontogenic infections, and six were the result of a retropharyngeal abscess [3]. These infections reach the mediastinum through loose connective tissue planes between the muscles in the neck. One major route is via the lateral pharyngeal space to the retrovisceral space [4]. In the case presented here, a retropharyngeal abscess developed into a deep neck abscess, tracking into the mediastinum and eroding into the thoracic and pericardial cavities within a few weeks. The organisms cultured from these locations were identical to those in the original retropharyngeal abscess. Although anaerobic organisms were not cultured from the abscess cavities, gas formation and foul smelling pus indicated their presence.

Bacteriological analysis of 36 previously reported cases revealed mixed aerobic and anaerobic organisms in 30 patients $(83 \%)$, and $\beta$-haemolytic streptococcus as the sole pathogen in 5 patients (14\%) [3]. We therefore used intravenous cefotiam and clindamycin to treat a mixed aerobic and anaerobic infection. Although antibiotics specific for E. cloacae, which was cultured from the pus, were administered systemically and used to irrigate the cervical abscess cavity, the abscess progressed and extended to the pericardial and pleural cavities. It has been reported that cervicomediastinal drainage and antibiotic therapy is insufficient in the management of DNM, once the abscess has extended to a level below the fourth thoracic vertebra or the tracheal bifurcation [2]. WHEATELY et al. [3] reported that transcervical mediastinal drainage provided incomplete or inadequate drainages in 26 (79\%) 
of the 33 patients with DNM. In the case presented here, cervicomediastinal drainage was indeed insufficient to treat the DNM. Whilst DNM has been successfully treated without extensive surgical drainage [5], aggressive drainage should be considered when managing life-threatening DNM. CT was found to be useful to assess the adequacy of treatment, and to determine the location of the abscess.

In conclusion, DNM is a very aggressive form of mediastinitis, with a mortality of approximately $40 \%$. Although the origins of DNM are in the oropharynx, this infection infrequently responds to cervical mediastinal drainage. The importance of proper mediastinal drainage, as well as the systemic administration of antibiotics specific for both aerobic and anaerobic organisms, must be emphasized. CT has been used to assess the adequacy of treatment.

\section{References}

1. Mukau L. Dissecting retropharyngeal abscess due to Fusobacterium necrophorum in an adult. South Med J 1985; 78: 476-478.

2. Estrera AS, Landay MJ, Grisham JM, Sinn DP, Platt MR. Descending necrotizing mediastinitis. Surg Gynecol Obstet 1983; 157: 545-552.

3. Wheatley MJ, Stirling MC, Kirsh MM, Gago O, Orringer MB. Descending necrotizing mediastinitis: transcervical drainage is not enough. Ann Thorac Surg 1990; 49: 780-784.

4. Moncada R, Warpeha R, Pikleman J, et al. Mediastinitis from odontogenic and deep cervical infection. Chest 1978; 73: 497-500.

5. de Marie S, Tjon A, Tham RTO, et al. Clinical infection and nonsurgical treatment of parapharyngeal space infections complicating throat infection. Rev Infect Dis 1989; 11: 975-982. 